\title{
Neural correlates of conversion disorder: overview and meta-analysis of neuroimaging studies on motor conversion disorder
}

\author{
Markus Boeckle ${ }^{1,2^{*}}$, Gregor Liegl ${ }^{1,3}$, Robert Jank ${ }^{1}$ and Christoph Pieh ${ }^{1,4}$
}

\begin{abstract}
Background: Conversion Disorders (CD) are prevalent functional disorders. Although the pathogenesis is still not completely understood, an interaction of genetic, neurobiological, and psychosocial factors is quite likely. The aim of this study is to provide a systematic overview on imaging studies on CDs and investigate neuronal areas involved in Motor Conversion Disorders (MCD).

Methods: A systematic literature search was conducted on CD. Subsequently a meta-analysis of functional neuroimaging studies on MCD was implemented using an Activation Likelihood Estimation (ALE). We calculated differences between patients and healthy controls as well as between affected versus unaffected sides in addition to an overall analysis in order to identify neuronal areas related to MCD.

Results: Patients with MCD differ from healthy controls in the amygdala, superior temporal lobe, retrosplenial area, primary motor cortex, insula, red nucleus, thalamus, anterior as well as dorsolateral prefrontal and frontal cortex. When comparing affected versus unaffected sides, temporal cortex, dorsal anterior cingulate cortex, supramarginal gyrus, dorsal temporal lobe, anterior insula, primary somatosensory cortex, superior frontal gyrus and anterior prefrontal as well as frontal cortex show significant differences.

Conclusions: Neuronal areas seem to be involved in the pathogenesis, maintenance or as a result of MCD. Areas that are important for motor-planning, motor-selection or autonomic response seem to be especially relevant. Our results support the emotional unawareness theory but also underline the need of more support by conduction imaging studies on both CD and MCD.
\end{abstract}

Keywords: Motor conversion disorder, Hysteria, Meta-analysis, Neuroimaging, Neurology

\section{Background}

In the recent Diagnostic and Statistical Manual of Mental Disorders (DSM-5; [1]), conversion disorder (CD) is defined as (1) having at least one symptom of altered voluntary motor or sensory function, (2) the presence of clinical findings supporting incompatibility between symptom and neurological or medical conditions, (3) the symptom is not better explained by another medical or

\footnotetext{
* Correspondence: markus.boeckle@donau-uni.ac.at

${ }^{1}$ Department of Psychotherapy and Biopsychosocial Health, Danube University Krems, Dr.-Karl-Dorrek-Str. 30, 3500 Krems, Austria

${ }^{2}$ Department of Cognitive Biology, University of Vienna, Vienna, Austria

Full list of author information is available at the end of the article
}

mental disorder, and (4) causes clinically significant distress or impairment. Due to problems of case definition and case ascertainment, prevalence rates vary largely [2]. However, $\mathrm{CDs}$ are not rare conditions, with prevalence rates of 1 to $3 \%$ in the general population [3, 4]. A prospective cohort study found that $5.6 \%$ of all outpatients have $\mathrm{CD}[5]$ and we suppose that treatment of patients with $\mathrm{CD}$ might be highly demanding similar to the treatment of patients with somatoform disorders [6].

Despite the historical relevance of the disorder in relation to hysteria, the current knowledge on aetiology and neurological background of CD is incomplete [7]. Similar to other psychiatric disorders an interrelation of 
genetic, neurobiological, and psychosocial factors is highly plausible. Twin studies showed that approximately $50 \%$ of the variance could be explained by genetic factors [8]. Dissociative symptoms are reported as a side effect of medication [9] and associated with endocrinological disorders [10], which points to neurobiological influences [7, 11]. Furthermore, psychosocial influences are assumed in the pathogenesis of dissociative disorders, which are according to ICD-10 [12] closely related to $\mathrm{CD}$. Psychological factors like alexithymia - the inability to identify and describe emotions in the self - is a risk factor for dissociative disorders [13]. There is rising evidence that dissociative symptoms are associated with trauma, as depersonalization and derealisation are quintessential responses to acute trauma [14] and dissociative symptoms often occur in patients with post-traumatic stress disorder (PTSD) [15].

Due to the relatedness of these disorders, similarities in the pathology might occur. For motor and somatosensory conversion Perez and colleagues [7] discuss different explanatory models of brain function: (1) disrupted inhibitory abilities with dysfunctionality in primary somatosensory and motor cortex [16-19] (2) modifications of the voluntary-intentional capacities with dysfunctionality in prefrontal areas [20, 21] (3) impaired attention based on dysfunctional anterior cingulate cortex, parietal associative cortex, striatum, thalamus [18, 22-24] (4) misconceptions of action authorship as a result of dysfunctionality in the tempoparietal junction, somatosensory cortex, anterior cingulate cortex, parietal associative cortex, and gyrus temporalis superior [25] (5) as well as affective disorders due to dysfunctionality in the amygdala and anterior cingulate cortex [25-27]. In addition to functional differences, structural changes have recently been discussed in conjunction with $\mathrm{CD}[28,29]$ whereby the premotor cortex, the primary motor cortex, and the cerebellum show changes in cortical thickness.

Although there is an incremental increase of knowledge about the causes of dissociative disorders, there is currently a lack of evidence for the effectiveness of psychological and pharmacological treatments. The International Society for the Study of Trauma and Dissociation noted in its guidelines that the treatment of Dissociative Identity Disorder is still in its infancy [14, 30]. For this reason, a recent study recommends further neurophysiological studies, including fMRI studies [31]. These studies should aim to not only provide more information about the aetiology of dissociative disorders, but also to identify at risk patients in a more timely manner and to thus treat dissociative conditions early and appropriately [31].

Even though numerous neuroimaging studies have been conducted on CD (e.g.: [2, 11, 32]) and neurobiological models have been proposed [7, 33], a meta- analytical approach is still missing. This might be due to the fact that different dissociative disorders, such as dissociative amnesia, fugue, dissociative identity disorder, motor and somatosensory conversions as well as pseudo-epileptic seizures might have various neurobiological correlates. We therefore provide as a first step a detailed list of publication on CD identified via a systematic literature research. However, because of potential differences in neurobiology between these disorders we mainly focused on motor conversion disorder (MCD) by conducting as a second step a meta-analytic approach on MCD using an activation likelihood estimation (ALE) to investigate neurobiological correlates of MCD.

\section{Methods}

\section{Literature search}

In order to identify the research articles relating to $C D$ that utilized neuro-imaging methods, we searched the following scientific databases: Medline, Psycinfo, Psyndex, and Cochrane. We did so using the following search terms: ("dissociative disorder" OR "functional disorder" OR "conversion disorder"), which simultaneously included the following neuro-imaging methods: ("neuro imaging" OR ("magnetic resonance imaging" OR ("magnetic" AND "resonance" AND "imaging") $O R$ "magnetic resonance imaging" OR "fMRI") OR ("magnetic resonance imaging" OR ("magnetic" AND "resonance" AND "imaging") OR "magnetic resonance imaging" OR "MRI") OR VBM OR PET). We included all published articles until August 2015. All titles and abstracts were independently rated by $\mathrm{MB}, \mathrm{GL}$, and RJ. All articles identified to include all given search terms by at least one of the raters were included in a subsequent full-text analysis. The criteria for inclusion in both steps (abstract and title analysis) were identical. The inclusion criteria were defined as the following: 1) paper written in English; 2) investigating human adults; 3) has to be primary research (thus excluding editorials, letters to the editor, systematic reviews, case studies, etc.); 4) study has to use one of the listed imaging methods (PET, MRI, SPECT); 5) studies investigated patients with CD or synonymous disorder according to DSM-IV, DSM-V, or ICD10 specifically excluding studies using hypnosis or feigning behaviour as alternative study population for CD. Any matches were included in the subsequent full text analysis process. All remaining articles were checked for accordance to the inclusion criteria. After analysing the full texts, we reported all studies using neuroimaging and $\mathrm{CD}$ or dissociative disorders as the classification of CD in ICD and DSM differ according to this point. As a next step we identified all studies about MCD that included imaging methods. For the inclusion in the meta-analysis, both neuro-imaging and MCD criterion had to be met and coordinates in MNI or Talairach space had to be provided. 


\section{Meta-analysis}

We conducted an Activation Likelihood Estimation (ALE) using GingerALE 2.3.1 [34-36], which supports the integration of multiple neuroimaging studies across imaging methods. Imaging studies based on MRI, PET, or SPECT and reporting Talairach or MNI coordinates including patients with MCD were included in the analysis. Coordinates published in MNI space were transformed into Talairach space using icbm2tal transformation [37, 38] provided by brainmap.org [39]. We incorporated all significant differences listed in the included papers. We used a Cluster-Level Analysis when analysing all experiments to correct for multiple identifications within one experiment as this procedure accommodates the spatially contiguous nature of the signal [36] and allows comparisons across different cognitive processes [35]. For the correction of multiple comparisons we used an uncorrected p-value of 0.001 as the cluster-forming threshold [40, 41], a cluster-level inference level of 0.05 with 1000 permutations, and a minimum cluster volume of $264 \mathrm{~mm}^{2}$. Threshold maps were viewed with Mango version 3.8 (772) [42], and significant clusters from the ALE analysis were superimposed on a standard anatomical image of the entire brain (Colin1.1.nii).

We analysed functional alterations in relation to MCD between patients and healthy controls for increased and decreased activation in patients separately. We calcualted additional subgroup meta-analyses in order to differentiate between increased and decreased activations of the affected versus unaffected sides of the brain. We excluded studies investigating the difference between dissociative disorders and hypnotized controls because of possible differences in neurobiological correlates.

The first analysis combined all reported functional imaging experiments within 12 articles. It includes 187 subjects, 73 foci and nine foci lying outside of the mask. In the subset analysis, in which greater activity in MCD patients versus healthy controls was analysed $(\mathrm{p}>\mathrm{c})$, is comprised of 148 subjects with 31 foci out of 6 studies, whereby four foci were lying outside of the mask. The sub analysis of patients showing lower activation than healthy controls $(\mathrm{p}<\mathrm{c})$ is based on four foci from two experiments and 36 individuals. The analysis of affected sides showing increased activation than the unaffected (a>ua) side and vice versa ( $\mathrm{a}<\mathrm{ua}$ ) is based on 27 foci from 4 experiments with 28 individuals and six foci from two experiments with 13 individual respectively. Low numbers of foci located outside the mask do not influence the results of the ALE analysis. Grey matters are reported within the nearest $+/-1 \mathrm{~mm}$. When recording the number of subjects in articles with multiple experiments, we used the lowest number of subjects reported in the respective article in order to calculate the most conservative ALE possible. Thus, when including the same study in different subsamples, samples from the same article can show varying numbers of subjects, which we statistically accounted for via cluster level analysis. Articles might report differing numbers of subjects dependent on the specific tests. We therefore used the lowest reported number of subjects that was listed for relevant subtests of one article, in order to calculate the most conservative influence of these areas. We used cluster-level analysis in order to adjust for the overlap of subjects reported from multiple tasks within one study.

\section{Results}

\section{Study selection}

The systematic literature search yielded 1035 results, whereby 266 duplicates were excluded; resulting in a total of 769 studies (Fig. 1). After a thorough analysis of the abstracts and titles (which led to the exclusion of 674 studies), 95 full texts were assessed to be potentially eligible for inclusion in this study. After full text analysis, the search resulted in 49 studies with neuroimaging of CD (Table 1).

\section{Study characteristics}

The systematic literature retrieval with the applied search terms came forth with 49 studies with neuroimaging of

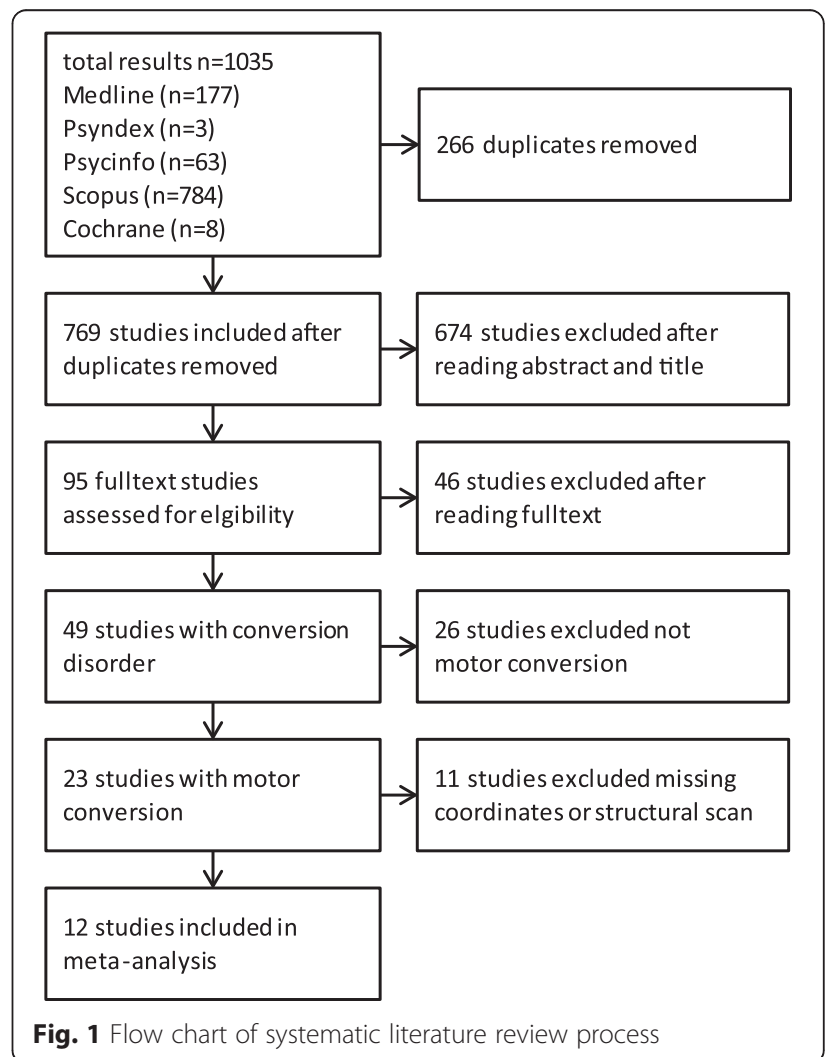


Table 1 All studies found with conversion disorder and neuroimaging. Studies indicated with ${ }^{\text {a }}$ were included in the meta-analysis

\begin{tabular}{|c|c|c|c|c|}
\hline Study & Disorder & Control group & Number of participants & Imaging method \\
\hline Atmaca, et al. [82] & motor conversion & yes & patients: $12(f)$, control: $12(f)$ & sMRI (1.5 Tesla) \\
\hline Atmaca, et al. [83] & somatization disorder & yes & patients: $20(f)$, control: $20(f)$ & sMRI (1.5 Tesla) \\
\hline Atmaca, et al. [84] & motor conversion & yes & patients: 20 (f), control: 20 (f) & sMRI (1.5 Tesla) \\
\hline Aybek, et al. [29] & motor conversion & yes & $\begin{array}{l}\text { patients: } 15(11 \mathrm{f}, 4 \mathrm{~m}) \text { [2 groups: } \\
\text { hemiparesis \& paraparesis], } \\
\text { control: } 25(16 \mathrm{f}, 9 \mathrm{~m})\end{array}$ & sMRI (3.0 Tesla) \\
\hline a Aybek, et al. [52] & motor conversion & yes & patients: 12 (9f, 3m), control 14 (11f, 3m) & fMRI (3.0 Tesla) \\
\hline aAybek, et al. [47] & motor conversion & yes & patients: 12 (8f, 4m), control 13 (10f, 3m) & fMRI (3.0 Tesla) \\
\hline Benbadis, et al. [85] & syncope of unknown origin & no & patients: $10(5 f, 5 \mathrm{~m})$ & $\begin{array}{l}\text { CT (7 patients), } \\
\text { MRI (8 patients) }\end{array}$ \\
\hline Blakemore, et al. [86] & motor conversion & yes & $\begin{array}{l}\text { patients: } 6 \text { (4f, } 2 \mathrm{~m}) \text {, feigner: } 12(8 \mathrm{f}), \\
\text { control: } 12(8 \mathrm{f})\end{array}$ & EEG \\
\hline Blakemore, et al. [87] & motor conversion & yes & $\begin{array}{l}\text { patients: } 6(4 f, 2 m) \text {, feigner: } 12(8 f), \\
\text { control: } 12(8 f)\end{array}$ & EEG \\
\hline Bonilha, et al. [88] & idiopathic dystonia & yes & patients: 7 (6f, 1m), control: $10(8 \mathrm{f}, 2 \mathrm{~m})$ & sMRI (3.0 Tesla) \\
\hline Burke, et al. [89] & sensory conversion & no & Patients: 10 (10f) & fMRI (3.0 Tesla) \\
\hline Burgmer, et al. [90] & motor conversion & yes & patients: 4 (m), control: $7(3 \mathrm{f}, 4 \mathrm{~m})$ & fMRI (3.0 Tesla) \\
\hline Carey, et al. [91] & body dysmorphic disorder & no & patients $6(4 \mathrm{~m}, 2 \mathrm{f})$ & SPECT (HMPAO) \\
\hline Cojan, et al. [19] & motor conversion & yes & $\begin{array}{l}\text { patients: } 1 \text { ( } f \text {, control: } 30 \\
\text { (normal:24, feigner: } 6)\end{array}$ & fMRI (1.5 Tesla) \\
\hline aczarnecki, et al. [60] & motor conversion & yes & patients:5 (3f, 2m) & $\begin{array}{l}\text { SPECT } \\
\text { (99mTc-Ethyl cysteinate) }\end{array}$ \\
\hline${ }^{a}$ de Lange, et al. [16] & motor conversion & no & patients: 8 (5f, 3m) & fMRI (1.5 Tesla) \\
\hline ade Lange, et al. [64] & motor conversion & no & patients:7 (5f, 2m) & fMRI (1.5 Tesla) \\
\hline de Lange, et al. [21] & motor conversion & no & patients: 8 & fMRI (1.5 Tesla) \\
\hline de Ruiter, et al. [92] & non clinical dissociative experiences & yes & $\begin{array}{l}\text { individuals: } 43 \text { (23 low }(15 f, 8 m) \text {, } \\
20 \text { high (10f, 10m) }\end{array}$ & fMRI (1.5 Tesla) \\
\hline Devinsky, et al. [93] & C-NES & yes & $\begin{array}{l}\text { C-NES only:22, C-NES + Epilepsy:38, } \\
\text { Epilepsy only: } 43, \\
\text { Epilepsy + other psych:59 }\end{array}$ & EEG \\
\hline aElzinga, et al. [46] & motor conversion & yes & patients: 13 (f), control:14 (f) & fMRI (1.5 Tesla) \\
\hline Felmingham, et al. [94] & dissociative PTSD & yes & patients:23 (13f, 10m), 12 dissociative & fMRI (1.5 Tesla) \\
\hline Feusner, et al. [95] & body dysmorphic disorder & yes & $\begin{array}{l}\text { patients: } 12(10 f, 2 \mathrm{~m}), \\
\text { control: } 13(11 \mathrm{f}, 2 \mathrm{~m})\end{array}$ & fMRI (3.0 Tesla) \\
\hline Feusner, et al. [96] & body dysmorphic disorder & yes & $\begin{array}{l}\text { patients: } 12(10 \mathrm{f}, 2 \mathrm{~m}), \\
\text { control: } 12(10 \mathrm{f}, 2 \mathrm{~m})\end{array}$ & sMRI (3.0 Tesla) \\
\hline Garcia-Campayo, et al. [97] & somatization disorder & no & patients: $11(5 f, 6 m)$ & $\begin{array}{l}\text { SPECT } \\
\text { (HMPAO or TC-bicisate) }\end{array}$ \\
\hline Ghaffar, et al. [62] & motor conversion & yes & patients: 3 (3f), control: 6 & fMRI (3.0 Tesla) \\
\hline Hakala, et al. [98] & somatization disorder & yes & patients: 10 (10f), control: 16 (16f) & sMRI (1.5 Tesla) \\
\hline Hoechstetter, et al. [99] & motor conversion & no & patients: 3 (2f, 1m) & MEG \\
\hline Hovorka, et al. [100] & PNES & no & patients: 56 (39f, 17m) & EEG \\
\hline Karatas, et al. [101] & PNES & no & patients: (88) & EEG \\
\hline Knyazeva, et al. [102] & PNES & yes & patients: 13 (8f, 5m), control: 13 (8f, 5m) & EEG \\
\hline Krüger, et al. [103] & dissociation DES & yes & patients: 50 & EEG \\
\hline Labate, et al. [28] & PNES & yes & $\begin{array}{l}\text { patients: } 20 \text { (11f, 9m), } \\
\text { control: } 40 \text { (21f, 19m) }\end{array}$ & sMRI (1.5 Tesla) \\
\hline Mailis-Gagnon, et al. [24] & hysterical anaesthesia & no & patients: 4 (3f, 1m) & fMRI (1.5 Tesla) \\
\hline Moser, et al. [104] & dissociation & yes & patients: 11 (11f), control: 9 (9f) & fMRI (3.0 Tesla) \\
\hline
\end{tabular}


Table 1 All studies found with conversion disorder and neuroimaging. Studies indicated with ${ }^{\text {a }}$ were included in the meta-analysis (Continued)

\begin{tabular}{|c|c|c|c|c|}
\hline Nicholson, et al. [105] & motor conversion & yes & $\begin{array}{l}\text { patients: } 15 \text { (10f, 5m) } \\
\text { control: } 31(19 f, 12 \mathrm{~m})\end{array}$ & sMRI (3.0 Tesla) \\
\hline Rauch, et al. [106] & body dysmorphic disorder & yes & patients: 8 (?) control: 8 (?) & MRI \\
\hline Roelofs, et al. [66] & motor conversion & no & patients: $6(f)$ & EEG \\
\hline Sar, et al. [107] & dissociative identity disorder & yes & patients: 21 (14f, $7 \mathrm{~m})$, control: $9(6 \mathrm{f}, 3 \mathrm{~m})$ & SPECT (HMPAO) \\
\hline aspence, et al. [20] & motor conversion & yes & patients: $2(\mathrm{~m})$, control: 6 & PET \\
\hline${ }^{a}$ Stone, et al. [74] & motor conversion & yes & patients: 4 (3f, 1m), control: 4 (3f, 1m) & fMRI (1.5 Tesla) \\
\hline avan Beilen, et al. [50] & motor conversion & yes & $\begin{array}{l}\text { patients: } 9 \text { (7f, } 2 \mathrm{~m}), \\
\text { control: } 21 \text { normal control } \\
(17 f, 4 m) 13 \text { feigning }(4 f, 9 m)\end{array}$ & fMRI (3.0 Tesla) \\
\hline van Der Kruijs, et al. [108] & PNES & yes & patients: $11(6 \mathrm{f}, 5 \mathrm{~m})$, control: $12(8 \mathrm{f}, 4 \mathrm{~m})$ & fMRI (3.0 Tesla) \\
\hline aVoon, et al. [43] & $\begin{array}{l}\text { conversion tremor, dystonia, } \\
\text { gait disorder }\end{array}$ & yes & patients: 11 (7f, 4m), control: $11(7 f, 4 m)$ & fMRI (1.5 Tesla) \\
\hline aVoon, et al. [27] & motor conversion & yes & $\begin{array}{l}\text { patients: } 16 \text { (10f, 6m), } \\
\text { control: } 16(10 \mathrm{f}, 6 \mathrm{~m})\end{array}$ & fMRI (1.5 Tesla) \\
\hline Voon, et al. [25] & motor conversion & no & patients: 8 (5f, 3m) & fMRI (1.5 Tesla) \\
\hline Vuilleumier, et al. [22] & sensorimotor conversion & no & patients: 7 (6f, 1m) & PET (HMPAO) \\
\hline Werring, et al. [109] & sensory conversion & yes & patients: 5 (4f, $1 \mathrm{~m})$, control 7 () & fMRI (1.5 Tesla) \\
\hline Yazici, et al. [110] & Astasia-Abasia & no & patients: 5 (3f, 2m) & PET (HMPAO) \\
\hline
\end{tabular}

Abbreviations: CT X-ray computed tomography; EEG electroencephalography; fMRI functional magnetic resonance tomography; sMRI structural magnetic resonance tomography; MEG magnetoencephalography; SPECT single-photon emission computed tomography; PET positron emission tomography, sex of individuals

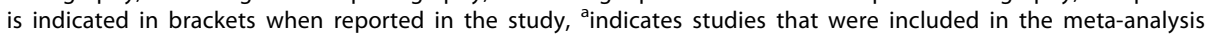

CD: 23 studies about MCD, 5 about psychogenic nonepileptic seizures, 4 about body dysmorphic disorder, 3 about somatization disorder, 3 about pure sensory conversion, and 11 studies about single disorders (for details see Table 1). All 49 studies either used MRI, CT, Spect, EEG, MEG, or PET as their imaging method. Out of all studies, 34 used a control group, whereby the others used withinsubject differences (Table 1). Of these 49 studies, 26 studies were excluded from the meta-analysis because they were not about MCD, 10 studies because of missing coordinates. We additionally excluded one study conducting structural scans, which resulted in 12 studies considered for meta-analyses. Out of the 23 included studies reporting imaging results of MCD, 16 studies used a control group (Table 2). Three studies looked at differences between affected and non-affected sides of the body (Table 2). All studies showed difference in (Table 2). The 12 studies that included MNI or Talairach coordinates, reported different affected areas listed in Table 3.

\section{Meta-analysis}

A cluster analysis for all experiments resulted in seven clusters (Table 4, Fig. 2), namely dorsolateral prefrontal cortex, amygdala, two clusters within the superior frontal gyrus, insula, frontal cortex as well as the dorsal anterior cingulate cortex. When calculating the subsample for $\mathrm{p}>\mathrm{c}, 13$ clusters were extracted within the following eight areas: amygdala, insula, retrosplenial area, superior temporal lobe, red nucleus, frontal cortex as well as anterior and dorsolateral prefrontal cortex (Fig. 3a). The analysis of $\mathrm{p}<\mathrm{c}$ resulted in two clusters in the primary motor cortex and thalamus. Activation in the a>ua resulted in seven areas with eight clusters: superior frontal gyrus, anterior prefrontal cortex, anterior insula, dorsal anterior cingulate cortex, dorsal temporal lobe, primary somatosensory cortex, and temporal cortex (Fig. 3b). The a<ua sample shows decreased activation in the frontal cortex and the supramarginal gyrus. For details see Table 2.

\section{Discussion}

The present study summarizes the results of functional brain-imaging (MRI, SPECT, and PET) studies on MCD via a meta-analytic approach. We found significant differential activation in several areas previously discussed in relation to $C D$. The current results of the metaanalysis suggest functional differences between patients with MCD and healthy controls in the amygdala, superior temporal lobe, retrosplenial area, primary motor cortex, insula, red nucleus, thalamus, anterior as well as dorsolateral prefrontal and frontal cortex (Fig. 2a). When comparing affected versus unaffected sides temporal cortex, dorsal anterior cingulate cortex, supramarginal gyrus, dorsal temporal lobe, anterior insula, primary somatosensory cortex, superior frontal gyrus and anterior as well as frontal cortex show significant differences 
Table 2 Neuroimaging studies on motor conversion disorders

\begin{tabular}{|c|c|c|c|}
\hline Citation & Type of conversion & Study design & Task \\
\hline Atmaca, et al. [82] & unilateral motor symptoms & matched control (healthy control) & structural differences \\
\hline Aybek, et al. [29] & conversion disorder with limb weakness & matched control (healthy control) & structural differences \\
\hline Aybek, et al. [52] & motor conversion disorder & matched control (healthy control) & visual stimuli - emotional faces \\
\hline Aybek, et al. [47] & motor conversion disorder & matched control (healthy control) & stressful memories task \\
\hline Blakemore, et al. [86] & unilateral upper limb conversion paresis & matched control (healthy control) & visual stimuli - reaction time task \\
\hline Burgmer, et al. [90] & dissociative paralysis in conversion disorders & matched controls & $\begin{array}{l}\text { movement execution and } \\
\text { observation-task }\end{array}$ \\
\hline Burke, et al. [89] & unilateral conversion disorder, sensory subtype & within group differences & vibrotactil stimulation \\
\hline \multirow[t]{3}{*}{ Czarnecki, et al. [60] } & psychogenic movement disorders & matched control (healthy control) & resting state \\
\hline & & & simple motor task \\
\hline & & & task vs. rest \\
\hline de Lange, et al. [16] & conversion paralysis & within subjects design & motor imagery task \\
\hline de Lange, et al. [64] & full or partial paralysis lateralized to one arm & within subjects design & motor imagery task \\
\hline de Lange, et al. [21] & $\begin{array}{l}\text { full or partial conversion paralysis } \\
\text { lateralized to one hand }\end{array}$ & within subject design & motor imagery task \\
\hline \multirow[t]{2}{*}{ Elzinga, et al. [46] } & dissociative disorder & matched control (healthy control) & working memory task effects \\
\hline & & & task load \\
\hline Ghaffar, et al. [62] & sensorimotor loss & case series & vibratory stimulation \\
\hline Nicholson, et al. [105] & motor conversion & matched control (healthy control) & structural \\
\hline Roelofs, et al. [66] & conversion paralysis & within subjects design & two-choice reaction task \\
\hline Spence, et al. [20] & motor conversion & matched control (healthy control) & movement execution \\
\hline Stone, et al. [74] & motor conversion & matched control (healthy control) & movement execution \\
\hline van Beilen, et al. [50] & motor conversion (paresis) & $\begin{array}{l}\text { Within subjects design, matched control } \\
\text { (healthy control) }\end{array}$ & $\begin{array}{l}\text { movement execution and } \\
\text { imagination }\end{array}$ \\
\hline Voon, et al. [27] & $\begin{array}{l}\text { conversion disorder with positive motor } \\
\text { symptoms }\end{array}$ & matched control (healthy control) & visual stimuli - emotional faces \\
\hline Voon, et al. [43] & $\begin{array}{l}\text { conversion disorder } \\
\text { (psychogenic movement disorder) }\end{array}$ & matched control (healthy control) & action selection task \\
\hline Vuilleumier, et al. [22] & unilateral hysterical sensorimotor loss & within subjects design & vibratory stimulation \\
\hline
\end{tabular}

(Fig. 2b). When analysing all functional experiments simultaneously dorsolateral prefrontal cortex, amygdala, superior frontal gyrus, insula, superior frontal gyrus, frontal cortex as well as dorsal anterior cingulate cortex show differential activation (Fig. 3).

\section{Patients increased activation in comparison to healthy control}

The largest area of the meta-analysis comparing patients to the control population is derived from two studies $[27,43]$. This cluster shows increased activity in the amygdala of patients with MCD in comparison to healthy controls. The amygdala is known to be involved in autonomic responses, including freezing behaviour, attention, vigilance and arousal [44]. Changes in this function might be an important factor for the occurrence of MCD, as patients with MCD show increased responses to startling responses $[43,45]$ as well as complications during the habituation to positive and negative emotional stimuli [27]. Additionally, increased functional activity in patients with MCD in comparison to healthy controls in the amygdala [27] might correlate with activity in the supplementary motor area (SMA). Voon et al. [43] suggest that the increased connectivity and activity in the SMAamygdala motor complex facilitates the expression of previously learned conversion motor representations. This aberrant activation of prefrontal areas is also supported by our study.

Prefrontal hyper-activity are involved in clusters 6, $8,11,12$, and 13, based on the frontal cortex (BA 9) $[46,47]$ anterior (BA 10) [47] as well as the dorsolateral prefrontal cortex (BA 46) [47]. Elzinga and colleagues [46] discuss the increased activation in the left anterior prefrontal cortex, left dorsolateral prefrontal cortex and left parietal lobe in correspondence to high loads and performance of the working memory in MCD patients. Aybek and colleagues [47] discuss that the increased activation in the dlPFC are 
Table 3 Affected areas and sides of studies included in the meta-analysis

\begin{tabular}{|c|c|c|}
\hline Citation & Area & Effect \\
\hline Aybek, et al. [52] & $\begin{array}{l}\text { Midbrain including periaqueductal grey area (bi), premotor } \\
\text { and supplementary areas (bi), dorsolateral prefrontal cortex (I), } \\
\text { cingulate cortex (I) superior frontal gyrus (I) }\end{array}$ & increased BOLD response \\
\hline \multirow[t]{2}{*}{ Aybek, et al. [47] } & $\begin{array}{l}\text { Supplementary motor area }(r) \text {, postcentral gyrus BA } 1(r) \text {, } \\
\text { postcentral gyrus BA4/3b }(r) \text {, superior temporal gyrus }(r) \text {, } \\
\text { angular gyrus at temporoparietal junction }(r) \text {, supramarginal } \\
\text { gyrus at temporoparietal junction (r) }\end{array}$ & increased BOLD response \\
\hline & lingual gyrus (I), parahippocampal gyrus (I), hippocampus (I) & decreased BOLD response \\
\hline \multirow[t]{2}{*}{ Czarnecki, et al. [60] } & $\begin{array}{l}\text { cerebellar hemispheres }(b i) \text {, superior orbital gyrus }(I) \text {, inferior } \\
\text { frontal gyrus }(I) \text {, insula }(I) \text {, precentral and postcentral gyri }(I) \text {, } \\
\text { supplementary motor area }(r) \text {, cerebellar hemisphere and } \\
\text { vermis (ipsi), supplementary motor area }(r)\end{array}$ & increased rCBF \\
\hline & $\begin{array}{l}\text { medial prefrontal cortex (bi), anterior cingulate cortex (I), } \\
\text { cerebellum (I), lingual gyrus (I) }\end{array}$ & reduced rCBF \\
\hline \multirow[t]{2}{*}{ de Lange, et al. [16] } & $\begin{array}{l}\text { dorsal intraparietal sulcus (r), dorsal precentral sulcus (bi), } \\
\text { posterior end of the Sylvian fissure }\end{array}$ & increased BOLD response to task complexity \\
\hline & $\begin{array}{l}\text { superior temporal cortex }(I) \text {, parietal operculum, prefrontal } \\
\text { cortex, superior temporal cortex }(r) \text {, posterior end of the } \\
\text { Sylvian fissure }\end{array}$ & increased BOLD response for the affected hand \\
\hline \multirow[t]{2}{*}{ de Lange, et al. [64] } & dorsal parietal and premotor cortex & increased BOLD response to task complexity \\
\hline & frontal cortex, gyrus rectus, superior temporal cortex & increased BOLD response for the affected hand \\
\hline Elzinga, et al. [46] & $\begin{array}{l}\text { anterior prefrontal cortex (I), dorsolateral prefrontal cortex (I), } \\
\text { parietal lobe (I) }\end{array}$ & increased $B O L D$ response \\
\hline Spence, et al. [20] & dorsolateral prefrontal cortex & reduced rCBF \\
\hline Stone, et al. [74] & $\begin{array}{l}\text { basal ganglia, insula, lingual gyri, interior frontal cortex, right } \\
\text { middle frontal gyrus (r), orbitofrontal cortex }\end{array}$ & increased BOLD response \\
\hline \multirow[t]{2}{*}{ van Beilen, et al. [50] } & $\begin{array}{l}\text { cingulate cortex (l), vental premotor cortex (ipsi), } \\
\text { supramarginal cortex (ipsi), superior temporal cortex (contra + } \\
\text { ipsi), anterior cingulate cortex (contra + ipsi), triangular cortex } \\
\text { inferior frontal (contra) }\end{array}$ & increased $B O L D$ response \\
\hline & $\begin{array}{l}\text { supramarginal gyrus (r), dIPFC (r), frontal pole (ipsi), ventral } \\
\text { lateral prefrontal (ipsi), precuneus (contra), cerebellum (ipsi) }\end{array}$ & decreased BOLD response \\
\hline \multirow[t]{2}{*}{ Voon, et al. [43] } & $\begin{array}{l}\text { anterior cingulate gyrus (I), primary motor cortex }(I) \text {, } \\
\text { somatosensory cortex }(I) \text {, secondary visual cortex }(r) \text {, ventral } \\
\text { premotor cortex (ipsi), supramarginal cortex (bi), anterior } \\
\text { cingulate cortex (contra), triangular cortex (contra) }\end{array}$ & increased $B O L D$ response \\
\hline & $\begin{array}{l}\text { primary motor cortex (r), somatosensory cortex (r), } \\
\text { dorsolateral prefrontal cortex (r), medial frontal pole (r), insular } \\
\text { cortex (I), cerebellum (l), frontal pole (ipsi), ventral lateral } \\
\text { prefrontal (ipsi), precuneus (contra), cerebellum (ipsi), } \\
\text { supplementary motor cortex (contra), frontal pole (contra), } \\
\text { ventrolateral prefrontal cortex (ipsi), orbitofrontal cortex (ipsi), } \\
\text { supramarginal cortex (contra), precuneus (contra), superior } \\
\text { parietal cortex (contra), frontal eye fields (contra) }\end{array}$ & decreased BOLD response \\
\hline \multirow[t]{2}{*}{ Voon, et al. [27] } & amygdala & increased BOLD response \\
\hline & amygdala to supplementary motor area & more connectivity $^{a}$ \\
\hline Vuilleumier, et al. [22] & Thalamus, caudate, putamen & decreased rCBF before treatment \\
\hline
\end{tabular}

l left, $r$ right, $b i$ both sides, dIPFC dorsolateral prefrontal cortex, $r C B F$ relative cerebral blood flow, BOLD blood oxygen level dependent. ${ }^{\text {a }}$ connectivity was measured as interregional correlation between conversion tremor and voluntary tremor within the same patients

based on active memory suppression during the recall of unwanted memories. Increased activation in patients with MCD was also reported during working memory studies in frontal cortex, anterior as well as dorsolateral prefrontal cortex [46]. The dorsolateral prefrontal cortex is also often dysfunctional in patients with other neuropsychiatric disorders that affect volition [20, 48, 49]. In MCD the top-down regulation of motor intention by prefrontal areas might have a crucial influence on its occurrence [43] as the decreased prefrontal activation might be correlated with impaired control of motor execution [50]. 
Table 4 Affected areas within the sample and subsamples

\begin{tabular}{|c|c|c|c|c|c|c|}
\hline Analysis & Cluster & Size & Center & Gray matter at center & \# of foci & Studies \\
\hline \multirow[t]{7}{*}{ all } & 1 & 456 & $44.7,36.8,26.5$ & BA 46: dIPFC & 3 & {$[47,50,74]$} \\
\hline & 2 & 448 & $19.6,-4.9,-10.5$ & Amygdala & 4 & {$[27,43]$} \\
\hline & 3 & 248 & $-12.1,51.9,38$ & BA 8: superior frontal gyrus & 2 & {$[16,64]$} \\
\hline & 4 & 192 & $-32.8,19.7,2$ & Insula & 1 & {$[16]$} \\
\hline & 5 & 192 & $-33,39.9,38.8$ & BA 8: superior frontal gyrus & 2 & {$[16,64]$} \\
\hline & 6 & 176 & $-43.1,34.6,31.7$ & BA 9: frontal cortex & 1 & {$[46]$} \\
\hline & 7 & 160 & $-6.3,15.3,37.7$ & BA 32: dorsal ACC & 1 & {$[52]$} \\
\hline \multirow[t]{13}{*}{$p>c$} & 1 & 544 & $19.7,-4.9,-10.6$ & Amygdala & 4 & {$[27,43]$} \\
\hline & 2 & 104 & $-32.5,19.1,2.2$ & Insula & 1 & [43] \\
\hline & 3 & 80 & $8,-49.2,8.8$ & BA 29: retrosplenial area & 1 & {$[43]$} \\
\hline & 4 & 72 & $-58.7,-48.5,2.4$ & BA 22: superior temporal lobe & 1 & {$[43]$} \\
\hline & 5 & 72 & $48.9,-45.8,10$ & BA 22: superior temporal lobe & 1 & [43] \\
\hline & 6 & 72 & $35.8,50,31.1$ & BA 9: frontal cortex & 1 & {$[47]$} \\
\hline & 7 & 64 & $-3,-22, .5$ & Red Nucleus & 1 & {$[52]$} \\
\hline & 8 & 64 & $23,41,5$ & BA 10: anterior PFC & 1 & {$[47]$} \\
\hline & 9 & 64 & $-7,-51,7$ & BA 29: retrosplenial area & 1 & [43] \\
\hline & 10 & 64 & $7.5,-48.7,21.3$ & BA 30: retrosplenial area & 1 & [43] \\
\hline & 11 & 64 & $47,37,27$ & BA 46: dIPFC & 1 & [47] \\
\hline & 12 & 64 & $-25,47,27$ & BA 9: frontal cortex & 1 & {$[46]$} \\
\hline & 13 & 64 & $-43,35,31$ & BA 9: frontal cortex & 1 & {$[46]$} \\
\hline \multirow[t]{2}{*}{$p<c$} & 1 & 752 & $-9.4,-11.9,69.7$ & BA 6: primary motor cortex & 1 & [43] \\
\hline & 2 & 728 & $-19.6,-20.2,2$ & Thalamus & 1 & [43] \\
\hline \multirow[t]{8}{*}{ a> ua } & 1 & 1080 & $-32.4,38.8,37.8$ & BA 8: superior frontal gyrus & 3 & {$[16,64]$} \\
\hline & 2 & 1008 & $-12.1,51.6,38.1$ & BA 8: superior frontal gyrus & 3 & {$[16,64]$} \\
\hline & 3 & 824 & $7.9,38,-12.3$ & BA 10: anterior PFC & 3 & {$[16,64]$} \\
\hline & 4 & 680 & $-54.8,-11.5,10.5$ & BA 43: anterior insula & 2 & {$[16,64]$} \\
\hline & 5 & 168 & $-8.6,13.6,43.6$ & BA 32: dorsal ACC & 1 & {$[50]$} \\
\hline & 6 & 128 & $62,-29,11.5$ & BA 42: dorsal temporal lobe & 1 & [16] \\
\hline & 7 & 128 & $-7.4,-40.6,58$ & BA 5: primary somatosensory cortex & 1 & [16] \\
\hline & 8 & 128 & $-49.2,-34.6,-3.9$ & BA 21: temporal cortex & 1 & {$[16]$} \\
\hline \multirow[t]{2}{*}{ a<ua } & 1 & 1336 & $40.8,35.4,26.9$ & BA 9: frontal cortex & 2 & {$[50,74]$} \\
\hline & 2 & 568 & $48.2,-43.3,42.4$ & BA 40: supramarginal gyrus & 1 & [50] \\
\hline
\end{tabular}

Size is represented in $\mathrm{mm}^{3}$. Clusters are described between two coordinates and its centre according to Talairach space. Each study contributing to the cluster is listed. Areas describe Brodmann areas (numbers), nearest grey matters, or nearest structures when no grey matter is within $+/-5 \mathrm{~mm}$. Number of underlying foci are reported for each cluster

Voon et al. [43] propose the increased activation of the insula in the context of potential motor-limbic network, whereby the insula is involved in the subjective representation of internal body and feeling states during motorselection. The increased activity of the insula found in our study might correlate with this limbic function as well as hyper-activity in the retrosplenial area, i.e. ventral posterior cingulate cortex represented in clusters 3,9 , and 10 in the analysis. This portion of the cingulate cortex is discussed to be important in the evaluation of emotional objects and memories of the past for self-relevance $[43,51]$.
In addition to these areas cluster 7 is centered at the red nucleus based on the study from Aybek and colleagus [52]. In their paper they do not confer the red nucleus as the most important area, but the periaqueductal grey (PAG), and hypothesize PAG to be a key region in the "freeze response" [52]. Especially the interaction between PAG and the amygdala seems to be important for autonomic fear responses and might via hyper activation result in a threat induced "freeze response" [52, 53].

The superior temporal area is another area represented in the found clusters [43]. This area incorporates the 


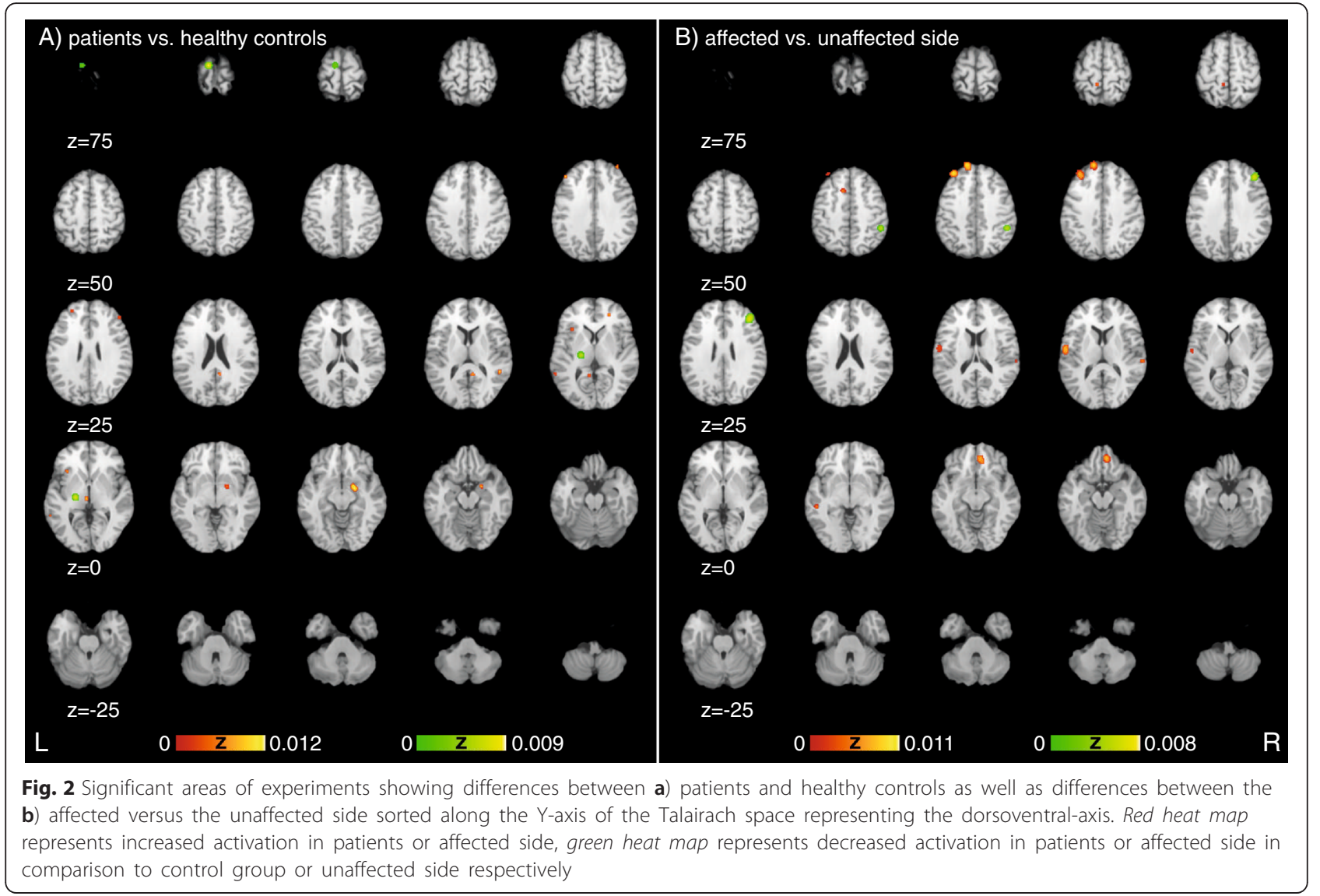

Broca area known for its contribution to language production and possibly understanding [54]. The difference between the superior temporal lobe activation in patients and controls might be based on differences in the processing of study instructions based on increased internal verbalizations of patients [55]. Additionally the temporal lobe is discussed to be a critical site in the network dealing with emotional trauma [56], whereby resolving or repressing emotional traumas seems to be partially mediated by temporal structures.

\section{Patients decreased activation in comparison to healthy control}

Our meta-analysis shows reduced activity in the thalamus [43]. Vuilleumier et al. [22] suggest that striatothalamocortical circuits controlling voluntary motor and sensorimotor conversion are crucial for functional disorders like conversion. Hypofunction of thalamus during conversion disorder resolved after recovery [22]. This effect might be based on the function of the thalamus as the main hub system to cortical areas from sensory and motor signals; thus it plays a crucial role in generating intentional movement and learning adaptive motor action $[22,57,58]$. Reduced activity of the primary motor cortex [43] supported by our results, might correlate with the mentioned hypo-activity of the thalamus. Additionally, it might also represent reduced motor activity during motor conversion disorder.

\section{Affected side increased activation in comparison to unaffected side}

The defect in motor action is additionally enhanced by problems with the anterior cingulate cortex (ACC; BA 32) found in cluster 5 when comparing increased activation of affected versus unaffected sides [50]. The ACC has one of its various functions in motor preparation, specifically selection of action, and conflict monitoring [59]. While Czarnecki et al. [60] report decreased activation in the ACC when comparing patients versus healthy controls, other studies report increased activation in MCD patients $[43,50,52]$. Similar to the hypothesis proposed by Voon et al. [43] in relation to increased activation of the amygdala, it might be that cingulate hyper-activation is related to emotional responses to motor action planning that inhibit motor execution especially when movement in the affected side is occurring. A study by van Beilen et al. [50] suggests that the over-activation in the cingulate cortex, especially when occurring in posterior parts, is related to alterations in functioning of the internal selection of movement that were previously described by Picard et al. 


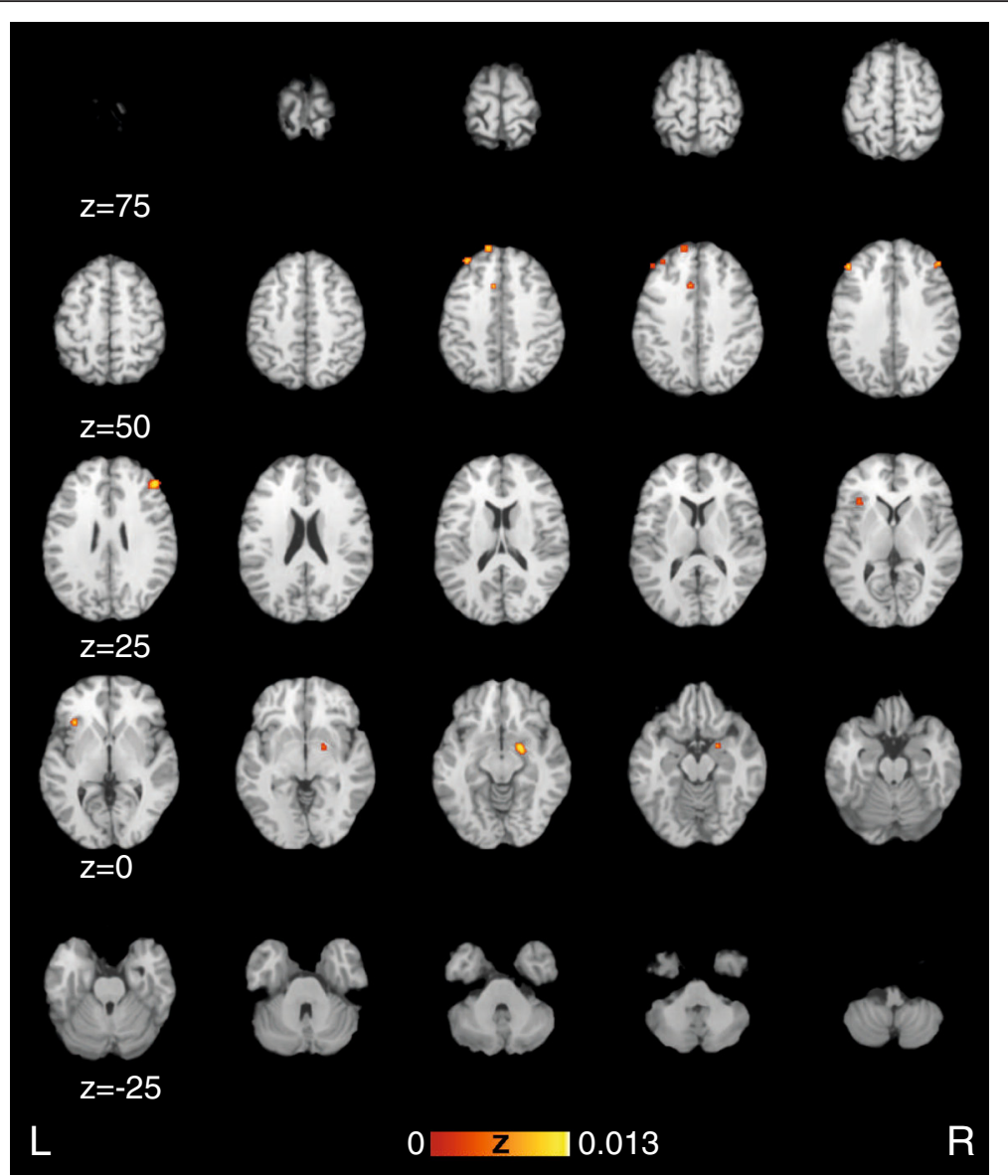

Fig. 3 Activation likelihood estimation maps showing significant clusters of all functional experiments and overlaid on the Colin Brain. Images are sorted along the $\mathrm{Y}$-axis of the Talairach space representing the dorso-ventral-axis

[61] and might be especially pronounced when comparing movements of the affected versus unaffected side.

Increased activation of the primary somatosensory cortex [16] of the affected side might be related to increased cognitions about motor planning and sensory input. Still, especially stimulation of the affected side was associated with a decrease of the primary somatosensory cortex in sensory conversion disorder $[62,63]$. Similarly to differences between patients and healthy controls, frontal and prefrontal areas are repeatedly identified within our metaanalysis when comparing increased activation in affected versus unaffected sides. The superior frontal gyrus $[16,64]$ and the anterior prefrontal cortex $[16,64]$ are significantly increased in the affected side. Increased prefrontal and frontal areas show increased activity in CP patients trying to move the affected body part $[16,17]$. The increase in activity due to motor preparation of the affected side seems also associated with increased self-monitoring [16, 64-66]. Studies reporting this difference are mainly based on studies on imagination of motor initiation.

The anterior insula was significantly increased in activity when the affected side was tested [16, 64]. The region, which is continuous with the primary gustatory cortex, is involved in the experience of emotions, particularly disgust [67-69]. Additionally, it is an important integrator of multimodal stimuli responsible for interfacing internal motivational states and external information. [7072]. Both functions seem to be increased in movement preparation in the affected compared to the unaffected side.

The dorsal temporal lobe [16] and the temporal cortex [16] are further areas showing increased activation. Similar to differences between patients and healthy controls, the temporal lobe may be involved in the network for dealing with emotional trauma, especially when resolving or repressing them [56]. The temporal region has furthermore been identified to be important for cognitive processes including implied and executed movement [73].

\section{Affected side decreased activation in comparison to unaffected side}

Decreases in the activity of medial prefrontal cortex for the affected side have been discussed to be based on impaired willed action [50,74]. This effect is contrary to 
other findings, where prefrontal activation is increased $[16,64]$. This difference might be task dependent, so that some tasks like motor imagination of the affected side result in increased activation of frontal areas, while others like movement execution show decreased activation of the affected side.

The supramarginal gyrus [50] exhibited decreased activation in the affected side compared to the unaffected side. The supramarginal gyrus has been revealed to be functionally coupled in conversion disorder with the dorsolateral prefrontal cortex [21]. This connection of the prefrontal area with the sensorimotor system are involved in generating and planning motor action [75]. The decreased activation might result in abnormal movement initiation processes [50].

\section{All experiments}

When all experiments are analysed and therefore differences between patients and healthy controls as well as differences between affected and unaffected sides are calculated within the same ALE irrespective of activation or deactivation, the following areas of interest were calculated, namely the dorsolateral and medial prefrontal cortex, the superior frontal gyrus, the insula as well as the amygdala and the dorsal anterior cingulate cortex. All areas have been identified in the sub analyses of the data. Even though no differentiation between activation or deactivation can be interpreted from the overall discussion, these areas seem to be repeatedly identified as dysfunctional in MCD and might be the core network for MCD.

\section{General discussion}

Our results show that emotional, motor planning, and inhibitory processes are involved in MCD. Instead of single miss-functioning of a specific neuroanatomical area, a complete network of areas seems to influence the presentation of MCD symptoms. Patients with MCD seem to primarily differentiate from healthy controls in the frontal and prefrontal cortices, ACC, and amygdala relevant for motor-planning and -selection, intentional behaviour, volition, and autonomic responses. This effect, as well as the results from all included experiments, is similar to the emotional unawareness theory of Perez and colleagues [7, 33], which states that the large-scale brain network mediates emotional and cognitive mechanisms and is modulated by experience-dependent neuroplasticity. Our meta-analysis gives strong indications and supports the differences proposed in ACC, prefrontal areas, the dlPFC, and the amygdala. Our meta-analysis does not show strong evidence for changes in the posterior parietal cortex as proposed by Perez et al. [7]. In the more recent discussion of functional unawareness by Perez et al. [33] all described areas are supported by our meta-analysis. Still, strong evidence is only supported for frontal and prefrontal areas, Insula, ACC, and amygdala. In order to be able to support the suggested functionalunawareness neural circuit framework more studies have to be conducted on MCD.

In summary, our results support the perspective that specific functions, which are discussed by Perez and colleagues [7, 33] are disrupted. We found substantive backing for the disturbance of intentional capacities with our analysis based on the repeated malfunctioning of frontal and prefrontal areas [20,21]. Changes in affective functions are highly plausible based on the increased activity of the amygdala and ACC in MCD [25-27]. Support of dysfunctional inhibitory abilities is provided by the increased activity of the affected side [16-19]. Attentional defects are partially substantiated by decreased activity of the thalamus in patients with $\mathrm{MCD}$, the reduced activity of the supramarginal gyrus and increased activity of the ACC in the affected side [18, 22-24]. Alterations of action authorship in MCD is partially supported by our meta-analysis based on the changes in tempoparietal junction, somatosensory cortex, ACC, parital cortex and the temporal lobe [18, 22-24]. When considering the overall analysis, most support is provided for changes in intentional and affective functions in patients with MCD. While conversion symptoms might correlate with failures of normal neurocognitive functioning, personal experience of patients of conversion symptoms perceived as disruptive, a loss of needed information, discontinuity of experience, or recurrent, jarring, involuntary intrusions into executive functioning and sense of self should not be lost sight of $[14,76]$.

There is growing evidence for a relation between dissociation and trauma: on the one hand dissociative symptoms often occur in patients with PTSD [15], on the other hand depersonalization and derealisation are quintessential responses to acute trauma [14]. For the dissociative subtype of PTSD, increased activation of frontal structures is consistent with hyper inhibition of those same limbic regions in states of pathological emotional overmodulation [77]. Studies support that a PTSD dissociative subtype should be included in DSM-5 [78]. It is not surprising that a meta-analysis of neuroimaging studies of PTSD patients [15] showed an overlap in neuronal activation with the current results of MCD patients. However, some of the abnormal activations in PTSD appear to be stress related, while other activations seem to be disease related [15]. This could also apply for CD; acute dissociation is primarily related to traumatic and/or overwhelming experiences, but dissociative symptoms in life-long presentations such as Dissociative Identity Disorder may also occur in circumstances that are unrelated to trauma or overwhelming circumstances [14]. Additionally, similarities to other functional disorders might exist $[79,80]$. 
Differences between resulting areas in the two subanalyses for patients versus healthy controls and affected versus unaffected side might be based on the fact that we calculate inter- and intra- individual differences. Still both comparisons shed light on the development and perpetuation of the disorder.

\section{Limitations}

This meta-analysis represents a first approach to combine the imaging results of MCD from various studies and is based on studies using differing imaging modalities and paradigms. Even though this approach might summarize various phenomena, the study of MCD is in need of a meta-analysis to more thoroughly examine the findings. The whole sample as well as the sub-analyses are sufficiently large enough to provide a first metaanalytical approach. Due to a low prevalence of MCD, the high costs of imaging, and the long data collection periods required, studies on MCD are rare. Ideally, a subcategory ALE analysis for each imaging modality and paradigm would be conducted. Due to the sparse imaging studies conducted on MCD, this was not feasible for the current study. Studies using hypnotization as a control are more prevalent, but might be explaining unintended phenomena within this meta-analysis and were therefore excluded. However, some of the mechanisms in hypnosis [81] and active feigning [19, 20] might share some neural mechanisms with MCD. Additionally, it is not clear whether the data presented in the various studies are based on recently developed conversions or on chronic conversion patients. Functional, as well as structural anatomy might change in the course of chronification. In the presented cases, not all patient characteristics are listed within the sample description, and medication as well as comorbidities might have skewed the data accordingly. Thus, it would be highly advantageous to understand functional and structural influences of the different disorders, as well as other factors influencing the variance, such as substance abuse, imaging parameters, software for analysis, experimental design etc. Future work should control for these differences, but at this time, the presented data can help present a starting point to the understanding of the neural correlates of MCD and CD in general. Still, our results are generalizable to limited extend on $\mathrm{CD}$ other than MCD. In order to better understand $C D$ and specific forms of $\mathrm{CD}$, more neurobiological research has to be conducted on disorders listed in Table 1, so that comparative ALEs can be calculated.

\section{Conclusions}

With this study, we are strengthening the evidence for neurobiological factors of MCD and hope to provide a first attempt at substantiating existing explanatory models based on literature reviews alone. We are attempting to advance the understanding of the aetiology and/or maintenance of MCD, which might serve as a basis for further research on this disorder.

\begin{abstract}
Abbreviations
ALE, activation likelihood estimation; CD, conversion disorder; MCD, motor conversion disorder; PTSD, post-traumatic symptom disorder; $\mathrm{FMRI}$, functional magnetic resonance tomography; VBM, voxel bases morphometry; PET, positron emission tomography; SPECT, single-photon emission computed tomography; CT, x-ray computed tomography; EEG, electroencephalography; MEG, magnetoencephalography; MNI, Montreal Neurological Institute and Hospital coordinate system; BA, Brodmann area; bi, bilateral; I, left; r, right; SMA, supplementary motor cortex; ACC, anterior cingulate cortex
\end{abstract}

\section{Acknowledgements}

We would like to thank our interns Marlene Schrimpf, Olivia Lackner, and Sophie Fuchsberger for helping in numerous ways during the research process. For supporting us with an initial abstract analysis form, we thank Prof. Gerald Gartlehner. We would also like to thank Doris Preininger and Stephanie Larumbe for the comments on the manuscript.

\section{Funding}

This study was co-funded by the Anniversary Fund of the City of Vienna for the Austrian Academy of Sciences, which is allocated for research projects on Cognitive Science named "Intentional Behavior in Ethology, Law, and Psychology: an Interdisciplinary Approach".

Availability of data and materials

All coordinates used in this study are publicly available in the original texts.

\section{Authors' contributions}

MB wrote the paper made substantial contributions to conception, design, data acquisition, review process, data analysis, and interpretation of the work. GL and RJ made substantial contributions to data acquisition and during review process. CP made substantial contributions to conception, design, review process, interpretation, and final approval. All authors have read and approve of the final version of the manuscript.

\section{Competing interests}

The authors declare that they have no competing interests.

\section{Consent to publish}

All coordinates used in this study are publicly available in the original texts.

\section{Ethics and consent to participate}

As a review and meta-analysis of previously reported studies no ethical approval or additional consent from participants is required.

\section{Author details}

${ }^{1}$ Department of Psychotherapy and Biopsychosocial Health, Danube University Krems, Dr.-Karl-Dorrek-Str. 30, 3500 Krems, Austria. ${ }^{2}$ Department of Cognitive Biology, University of Vienna, Vienna, Austria. ${ }^{3}$ Medical Clinic, Department of Psychosomatic Medicine, Charité - Universitätsmedizin, Berlin, Germany. ${ }^{4}$ Department of Psychosomatic Medicine, University Hospital Regensburg, Regensburg, Germany.

Received: 7 December 2015 Accepted: 27 May 2016

Published online: 10 June 2016

\section{References}

1. American Psychiatric Association. Diagnostic and statistical manual of mental disorders (5th ed.). Washington, DC: Author; 2013.

2. Carson AJ, Brown R, David AS, Duncan R, Edwards MJ, Goldstein LH, Grunewald R, Howlett S, Kanaan R, Mellers J, et al. Functional (conversion) neurological symptoms: research since the millennium. J Neurol Neurosurg Psychiatry. 2012;83(8):842-50.

3. Johnson JG, Cohen P, Kasen S, Brook JS. Dissociative disorders among adults in the community, impaired functioning, and axis I and II comorbidity. J Psychiatr Res. 2006;40(2):131-40. 
4. Şar V, Akyüz G, Doğan O. Prevalence of dissociative disorders among women in the general population. Psychiatry Res. 2007;149(1-3):169-76.

5. Stone J, Carson A, Duncan R, Roberts R, Warlow C, Hibberd C, Coleman R, Cull R, Murray G, Pelosi A, et al. Who is referred to neurology clinics?-The diagnoses made in 3781 new patients. Clin Neurol Neurosurg. 2010;112(9):747-51.

6. Boeckle M, Liegl G, Leitner A, Pieh C. How burdensome is the treatment of patients with somatoform disorders? Z Psychosom Med Psychother. 2014;60(4):383-91.

7. Perez DL, Barsky AJ, Daffner K, Silbersweig DA. Motor and somatosensory conversion disorder: a functional unawareness syndrome? J Neuropsychiatry Clin Neurosci. 2012;24(2):141-51.

8. Jang KL, Paris J, Zweig-Frank H, Livesley WJ. Twin study of dissociative experience. J Nerv Ment Dis. 1998;186(6):345-51.

9. Feng Z, Huang J, Xu Y, Zhang M, Hu S. Dissociative disorder induced by clarithromycin combined with rabeprazole in a patient with gastritis. J Int Med Res. 2013;41(1):239-43.

10. Mizutani K, Nishimura K, Ichihara A, Ishigooka J. Dissociative disorde due to Graves' hyperthyroidism: a case report. Gen Hosp Psychiatry. 2014;36(4):450.e451-2.

11. Atmaca M. Neuroimaging in somatoform disorder: a review. Turkish Journal of Psychiatry. 2012;23(4):276-81

12. AMA, Association AM: ICD-10-CM 2015: The Complete Official Codebook: Amer Medical Assn; 2014.

13. Modestin J, Lötscher K, Erni T. Dissociative experiences and their correlates in young non-patients. Psychol Psychother Theory Res Pract. 2002;75(1):53-64.

14. Spiegel D, Loewenstein RJ, Lewis-Fernández R, Sar V, Simeon D, Vermetten E, Cardeña E, Dell PF. Dissociative disorders in DSM-5. Depress Anxiety. 2011;28(12):E17-45.

15. Li L, Wu M, Liao Y, Ouyang L, Du M, Lei D, Chen L, Yao L, Huang X, Gong Q. Grey matter reduction associated with posttraumatic stress disorder and traumatic stress. Neurosci Biobehav Rev. 2014;43:163-72.

16. de Lange FP, Roelofs $K$, Toni I. Increased self-monitoring during imagined movements in conversion paralysis. Neuropsychologia. 2007;45(9):2051-8.

17. Marshall JC, Halligan PW, Fink GR, Wade DT, Frackowiak RS. The functional anatomy of a hysterical paralysis. Cognition. 1997;64(1):B1-8.

18. Tiihonen J, Kuikka J, Viinamaki H, Lehtonen J, Partanen J. Altered cerebral blood flow during hysterical paresthesia. Biol Psychiatry. 1995;37(2):134-5.

19. Cojan Y, Waber L, Carruzzo A, Vuilleumier P. Motor inhibition in hysterical conversion paralysis. Neurolmage. 2009;47(3):1026-37.

20. Spence SA, Crimlisk HL, Cope H, Ron MA, Grasby PM. Discrete neurophysiological correlates in prefrontal cortex during hysterical and feigned disorder of movement. Lancet. 2000;355(9211):1243-4.

21. de Lange FP, Toni I, Roelofs K. Altered connectivity between prefrontal and sensorimotor cortex in conversion paralysis. Neuropsychologia. 2010;48(6):1782-8

22. Vuilleumier P, Chicherio C, Assal F, Schwartz S, Slosman D, Landis T. Functional neuronantomical correlates of hysterical sensorimotor loss. Brain. 2001;124:1077-90.

23. Saj A, Arzy $S$, Vuilleumier $P$. Functional brain imaging in a woman with spatial neglect due to conversion disorder. JAMA. 2009;302(23):2552-4.

24. Mailis-Gagnon A, Giannoylis I, Downar J, Kwan CL, Mikulis DJ, Crawley AP, Nicholson K, Davis KD. Altered central somatosensory processing in chronic pain patients with "hysterical" anesthesia. Neurology. 2003;60(9):1501-7.

25. Voon V, Gallea C, Hattori N, Bruno M, Ekanayake V, Hallett M. The involuntary nature of conversion disorder. Neurology. 2010;74(3):223-8.

26. Kanaan RA, Craig TK, Wessely SC, David AS. Imaging repressed memories in motor conversion disorder. Psychosom Med. 2007;69(2):202-5.

27. Voon V, Brezing C, Gallea C, Ameli R, Roelofs K, LaFrance Jr WC, Hallett M. Emotional stimuli and motor conversion disorder. Brain. 2010;133(Pt 5):1526-36.

28. Labate A, Cerasa A, Mula M, Mumoli L, Gioia MC, Aguglia U, Quattrone A, Gambardella A. Neuroanatomic correlates of psychogenic nonepileptic seizures: a cortical thickness and VBM study. Epilepsia. 2012;53(2):377-85.

29. Aybek S, Nicholson TR, Draganski B, Daly E, Murphy DG, David AS, Kanaan RA. Grey matter changes in motor conversion disorder. J Neurol Neurosurg Psychiatry. 2014;85(2):236-8.
30. Dissociation ISftSoTa. Guidelines for treating dissociative identity disorder in adults, third revision. J Trauma Dissociation. 2011;12(2):115-87.

31. van der Kruijs SJM, Bodde NMG, Carrette E, Lazeron RHC, Vonck KEJ, Boon PAJM, Langereis GR, Cluitmans PJM, Feijs LMG, Hofman PAM, et al. Neurophysiological correlates of dissociative symptoms. J Neurol Neurosurg Psychiatry. 2014;85(2):174-9.

32. Feinstein A. Conversion disorder: advances in our understanding. CMAJ. 2011;183(8):915-20.

33. Perez DL, Dworetzky BA, Dickerson BC, Leung L, Cohn R, Baslet G, Silbersweig DA. An integrative neurocircuit perspective on psychogenic nonepileptic seizures and functional movement disorders: neural functional unawareness. Clin EEG Neurosci. 2015;46(1):4.

34. Eickhoff SB, Laird AR, Grefkes C, Wang LE, Zilles K, Fox PT. Coordinate-based activation likelihood estimation meta-analysis of neuroimaging data: a random-effects approach based on empirical estimates of spatial uncertainty. Hum Brain Mapp. 2009;30(9):2907-26.

35. Turkeltaub PE, Eickhoff SB, Laird AR, Fox M, Wiener M, Fox P. Minimizing within-experiment and within-group effects in activation likelihood estimation meta-analyses. Hum Brain Mapp. 2012:33(1):1-13.

36. Eickhoff SB, Bzdok D, Laird AR, Kurth F, Fox PT. Activation likelihood estimation meta-analysis revisited. Neurolmage. 2012:59(3):2349-61.

37. Lancaster JL, Tordesillas-Gutiérrez D, Martinez M, Salinas F, Evans A, Zilles K, Mazziotta JC, Fox PT. Bias between MNI and Talairach coordinates analyzed using the ICBM-152 brain template. Hum Brain Mapp. 2007;28(11):1194-205.

38. Laird AR, Robinson JL, McMillan KM, Tordesillas-Gutiérrez D, Moran ST, Gonzales SM, Ray KL, Franklin C, Glahn DC, Fox PT, et al. Comparison of the disparity between Talairach and MNI coordinates in functional neuroimaging data: validation of the Lancaster transform. Neurolmage. 2010;51(2):677-83.

39. brainmap.org [http://www.brainmap.org/index.html]. Accessed 1 May 2016.

40. Laird AR, Fox PM, Price CJ, Glahn DC, Uecker AM, Lancaster JL, Turkeltaub PE, Kochunov P, Fox PT. ALE meta-analysis: controlling the false discovery rate and performing statistical contrasts. Hum Brain Mapp. 2005;25(1):155-64.

41. Genovese CR, Lazar NA, Nichols T. Thresholding of statistical maps in functional neuroimaging using the false discovery rate. Neurolmage. 2002; 15(4):870-8

42. Res Imaging Inst website [http://rii.uthscsa.edu/mango/]. Accessed 1 May 2016.

43. Voon V, Brezing C, Gallea C, Hallett M. Aberrant supplementary motor complex and limbic activity during motor preparation in motor conversion disorder. Mov Disord. 2011;26(13):2396-403.

44. Lang PJ, Davis M. Emotion, motivation, and the brain: reflex foundations in animal and human research. Prog Brain Res. 2006;156:3-29.

45. Seignourel PJ, Miller K, Kellison I, Rodriguez R, Fernandez HH, Bauer RM, Bowers D, Okun MS. Abnormal affective startle modulation in individuals with psychogenical movement disorder. Mov Disord. 2007;22(9):1265-71.

46. Elzinga BM, Ardon AM, Heijnis MK, De Ruiter MB, Van Dyck R, Veltman DJ. Neural correlates of enhanced working-memory performance in dissociative disorder: a functional MRI study. Psychol Med. 2007;37(2):235-45.

47. Aybek S, Nicholson TR, Zelaya F, O'Daly OG, Craig TJ, David AS, Kanaan RA. Neural correlates of recall of life events in conversion disorder. JAMA Psychiatry. 2014;71(1):52-60.

48. Spence SA. Hysterical paralyses as disorders of action. Cogn Neuropsychiatry. 1999;4(3):203-26.

49. Spence SA, Hirsch SR, Brooks DJ, Grasby PM. Prefrontal cortex activity in people with schizophrenia and control subjects. Evidence from positron emission tomography for remission of 'hypofrontality'with recovery from acute schizophrenia. Br J Psychiatry. 1998;172(4):316-23.

50. van Beilen M, de Jong BM, Gieteling EW, Renken R, Leenders KL. Abnormal parietal function in conversion paresis. PLoS One. 2011;6(10), e25918.

51. Vogt BA, Vogt L, Laureys S. Cytology and functionally correlated circuits of human posterior cingulate areas. Neurolmage. 2006;29(2):452-66.

52. Aybek S, Nicholson TR, O'Daly O, Zelaya F, Kanaan RA, et al. Emotion-Motion Interactions inConversion Disorder: An fMRI Study. PLoS ONE. 2015;10(4): e0123273. doi: 10.1371/journal.pone.0123273.

53. Myers B, Dolgas CM, Kasckow J, Cullinan WE, Herman JP. Central stressintegrative circuits: forebrain glutamatergic and GABAergic projections to the dorsomedial hypothalamus, medial preoptic area, and bed nucleus of the stria terminalis. Brain Struct Funct. 2014;219(4):1287-303.

54. Hinke RM, Hu X, Stillman AE, Kim S-G, Merkle H, Salmi R, Ugurbil K. Functional magnetic resonance imaging of Broca's area during internal speech. Neuroreport. 1993;4(6):675-8. 
55. Rutten G, Van Rijen P, Van Veelen C, Ramsey N. Language area localization with three-dimensional functional magnetic resonance imaging matches intrasulcal electrostimulation in Broca's area. Ann Neurol. 1999;46(3):405-8.

56. Naga AA, Devinsky O, Barr WB. Somatoform disorders after temporal lobectomy. Cogn Behav Neurol. 2004;17(2):57-61.

57. Graybiel AM, Aosaki T, Flaherty AW, Kimura M. The basal ganglia and adaptive motor control. Science. 1994;265(5180):1826-31.

58. Steriade M, Llinás RR. The functional states of the thalamus and the associated neuronal interplay. Physiol Rev. 1988;68(3):649-742.

59. Botvinick M, Nystrom LE, Fissell K, Carter CS, Cohen JD. Conflict monitoring versus selection-for-action in anterior cingulate cortex. Nature. 1999;402(6758):179-81.

60. Czarnecki K, Jones DT, Burnett MS, Mullan B, Matsumoto JY. SPECT perfusion patterns distinguish psychogenic from essential tremor. Parkinsonism Relat Disord. 2011;17(5):328-32.

61. Picard N, Strick PL. Motor areas of the medial wall: a review of their location and functional activation. Cereb Cortex. 1996;6(3):342-53.

62. Ghaffar O, Staines WR, Feinstein A. Unexplained neurologic symptoms: an fMRI study of sensory conversion disorder. Neurology. 2006:67(11):2036-8.

63. Bell V, Oakley DA, Halligan PW, Deeley Q. Dissociation in hysteria and hypnosis: evidence from cognitive neuroscience. J Neurol Neurosurg Psychiatry. 2011;82(3):332-9.

64. de Lange FP, Roelofs K, Toni I. Motor imagery: a window into the mechanisms and alterations of the motor system. Cortex. 2008;44(5):494-506.

65. Vuilleumier P. Hysterical conversion and brain function. Prog Brain Res 2005;150:309-29.

66. Roelofs K, de Bruijn ER, Van Galen GP. Hyperactive action monitoring during motor-initiation in conversion paralysis: an event-related potential study. Biol Psychol. 2006;71(3):316-25.

67. Deen B, Pitskel NB, Pelphrey KA. Three systems of insular functional connectivity identified with cluster analysis. Cereb Cortex. 2010;21(7):14981506.

68. Calder AJ, Keane J, Manes F, Antoun N, Young AW. Impaired recognition and experience of disgust following brain injury. Nat Neurosci. 2000;3(11):1077-8.

69. Adolphs R, Tranel D, Damasio AR. Dissociable neural systems for recognizing emotions. Brain Cogn. 2003;52(1):61-9.

70. Mesulam MM, Mufson EJ. Insula of the old world monkey. III: efferent cortical output and comments on function. J Comp Neurol. 1982;212(1):38-52

71. Craig AD. How do you feel? Interoception: the sense of the physiological condition of the body. Nat Rev Neurosci. 2002;3(8):655-66.

72. Craig AD. How do you feel-now? The anterior insula and human awareness. Nat Rev Neurosci. 2009;10(1):59-70.

73. Allison T, Puce A, McCarthy G. Social perception from visual cues: role of the STS region. Trends Cogn Sci. 2000;4(7):267-78.

74. Stone J, Zeman A, Simonotto E, Meyer M, Azuma R, Flett S, Sharpe M. FMRI in patients with motor conversion symptoms and controls with simulated weakness. Psychosom Med. 2007;69(9):961-9.

75. Frith $C D$, Wolpert DM. Abnormalities in the awareness and control of action Philos Trans R Soc Lond B. 2000;355(1404):1771-88.

76. Dell P. The phenomena of pathological dissociation. In: Dissociation and the dissociative disorders: DSM-V and beyond. New York: Routledge; 2009. p. 225-38.

77. Lanius RA, Vermetten E, Loewenstein RJ, Brand B, Schmahl C, Bremner JD, Spiegel D. Emotion modulation in PTSD: Clinical and neurobiological evidence for a dissociative subtype. Am J Psychiatry. 2010;167(6):640-7.

78. Blevins CA, Weathers FW, Witte TK. Dissociation and posttraumatic stress disorder: a latent profile analysis. J Trauma Stress. 2014;27(4):388-96.

79. Boeckle M, Schrimpf M, Liegl G, Pieh C. Neural correlates of somatoform disorders from a meta-analytic perspective on neuroimaging studies. Neurolmage: Clinical. 2016. doi:10.1016/j.nicl.2016.1004.1001.

80. Bourke JH, Langford RM, White PD. The common link between functional somatic syndromes may be central sensitisation. J Psychosom Res. 2015; 78(3):228-36.

81. Halligan PW, Athwal BS, Oakley DA, Frackowiak RS. Imaging hypnotic paralysis: implications for conversion hysteria. Lancet. 2000;355(9208):986-7.
82. Atmaca M, Aydin A, Tezcan E, Poyraz AK, Kara B. Volumetric investigation of brain regions in patients with conversion disorder. Prog Neuropsychopharmacol Biol Psychiatry. 2006;30(4):708-13.

83. Atmaca M, Sirlier B, Yildirim H, Kayali A. Hippocampus and amygdalar volumes in patients with somatization disorder. Prog NeuroPsychopharmacol Biol Psychiatry. 2011;35(7):1699-703.

84. Atmaca M, Baykara S, Mermi O, Yildirim H, Akaslan U. Pituitary volumes are changed in patients with conversion disorder. Brain Imaging Behav. 2015;10(1):92-5.

85. Benbadis SR, Chichkova R. Psychogenic pseudosyncope: an underestimated and provable diagnosis. Epilepsy Behav. 2006;9(1):106-10.

86. Blakemore RL, Hyland BI, Hammond-Tooke GD, Anson JG. Distinct modulation of event-related potentials during motor preparation in patients with motor conversion disorder. PLoS One. 2013;8(4), e62539.

87. Blakemore RL, Hyland BI, Hammond-Tooke GD, Anson JG. Deficit in latestage contingent negative variation provides evidence for disrupted movement preparation in patients with conversion paresis. Biol Psychol. 2015;109:73-85.

88. Bonilha L, de Vries PM, Vincent DJ, Rorden C, Morgan PS, Hurd MW Besenski N, Bergmann KJ, Hinson VK. Structural white matter abnormalities in patients with idiopathic dystonia. Mov Disord. 2007:22(8):1110-6.

89. Burke MJ, Ghaffar O, Staines WR, Downar J, Feinstein A. Functional neuroimaging of conversion disorder: the role of ancillary activation. Neurolmage Clinical. 2014;6:333-9.

90. Burgmer $M$, Konrad C, Jansen A, Kugel H, Sommer J, Heindel W, Ringelstein EB, Heuft G, Knecht S. Abnormal brain activation during movement observation in patients with conversion paralysis. Neurolmage. 2006;29(4):1336-43.

91. Carey P, Seedat S, Warwick J, van Heerden B, Stein DJ. SPECT imaging of body dysmorphic disorder. J Neuropsychiatry Clin Neurosci. 2004;16(3):357-9.

92. de Ruiter MB, Veltman DJ, Phaf RH, van Dyck R. Negative words enhance recognition in nonclinical high dissociators: an fMRI study. Neurolmage. 2007;37(1):323-34

93. Devinsky O, Mesad S, Alper K. Nondominant hemisphere lesions and conversion nonepileptic seizures. J Neuropsychiatry Clin Neurosci. 2001;13(3):367-73

94. Felmingham K, Kemp AH, Williams L, Falconer E, Olivieri G, Peduto A, Bryant $R$. Dissociative responses to conscious and non-conscious fear impact underlying brain function in post-traumatic stress disorder. Psychol Med. 2008;38(12):1771-80.

95. Feusner JD, Townsend J, Bystritsky A, Bookheimer S. Visual information processing of faces in body dysmorphic disorder. Arch Gen Psychiatry. 2007;64(12):1417-25

96. Feusner JD, Townsend J, Bystritsky A, McKinley M, Moller H, Bookheimer S. Regional brain volumes and symptom severity in body dysmorphic disorder. Psychiatry Res Neuroimaging. 2009;172(2):161-7.

97. Garcia-Campayo J, Sanz-Carrillo C, Baringo T, Ceballos C. SPECT scan in somatization disorder patients: an exploratory study of eleven cases. Aust N Z J Psychiatry. 2001;35(3):359-63.

98. Hakala M, Karlsson H, Kurki T, Aalto S, Koponen S, Vahlberg T, Niemi PM. Volumes of the caudate nuclei in women with somatization disorder and healthy women. Psychiatry Res Neuroimaging. 2004;131(1):71-8.

99. Hoechstetter K, Meinck HM, Henningsen P, Scherg M, Rupp A. Psychogenic sensory loss: magnetic source imaging reveals normal tactile evoked activity of the human primary and secondary somatosensory cortex. Neurosci Lett. 2002;323(2):137-40.

100. Hovorka J, Nezadal T, Herman E, Nemcova I, Bajacek M. Psychogenic nonepileptic seizures, prospective clinical experience: diagnosis, clinical features, risk factors, psychiatric comorbidity, treatment outcome. Epileptic Disord. 2007;9 Suppl 1:S52-8.

101. Karatas H, Tezer FI, Dericioglu N, Saygi S. Pitfalls of psychogenic nonepileptic status epilepticus. Brain Impairment. 2012;13(3):333-8.

102. Knyazeva MG, Jalili M, Frackowiak RS, Rossetti AO. Psychogenic seizures and frontal disconnection: EEG synchronisation study. J Neurol Neurosurg Psychiatry. 2011;82(5):505-11.

103. Krüger $C$, Bartel $P$, Fletcher $L$. Dissociative mental states are canonically associated with decreased temporal theta activity on spectral analysis of EEG. J Trauma Dissociation. 2013;14(4):473-91.

104. Moser DA, Aue T, Wang ZS, Serpa SR, Favez N, Peterson BS, Schechter DS. Limbic brain responses in mothers with post-traumatic stress disorder and comorbid dissociation to video clips of their children. 
Stress-the International Journal on the Biology of Stress. 2013;16(5):493-502.

105. Nicholson TR, Aybek S, Kempton MJ, Daly EM, Murphy DG, David AS, Kanaan RA. A structural MRI study of motor conversion disorder: evidence of reduction in thalamic volume. J Neurol Neurosurg Psychiatry. 2013;85(2): 227-9.

106. Rauch SL, Phillips KA, Segal E, Makris N, Shin LM, Whalen PJ, Jenike MA, Caviness VS, Jr., Kennedy DN. A preliminary morphometric magnetic resonance imaging study of regional brain volumes in body dysmorphic disorder. Psychiatry Res Neuroimaging. 2003;122(1):13-9.

107. Sar V, Unal SN, Ozturk E. Frontal and occipital perfusion changes in dissociative identity disorder. Psychiatry Res. 2007;156(3):217-23.

108. van Der Kruijs SJM, Bodde NMG, Vaessen MJ, Lazeron RHC, Vonck K, Boon P, Hofman PAM, Backes WH, Aldenkamp AP, Jansen JFA. Functional connectivity of dissociation in patients with psychogenic non-epileptic seizures. J Neurol Neurosurg Psychiatry. 2012;83(3):239-47.

109. Werring DJ, Weston L, Bullmore ET, Plant GT, Ron MA. Functional magnetic resonance imaging of the cerebral response to visual stimulation in medically unexplained visual loss. Psychol Med. 2004;34(4):583-9.

110. Yazici KM, Kostakoglu L. Cerebral blood flow changes in patients with conversion disorder. Psychiatry Res. 1998;83(3):163-8.

\section{Submit your next manuscript to BioMed Central} and we will help you at every step:

- We accept pre-submission inquiries

- Our selector tool helps you to find the most relevant journal

- We provide round the clock customer support

- Convenient online submission

- Thorough peer review

- Inclusion in PubMed and all major indexing services

- Maximum visibility for your research

Submit your manuscript at www.biomedcentral.com/submit 